\title{
The high density region of QCD in a large mass and chemical potential model
}

\author{
Roberto De Pietri and Alessandra Feo* \\ Dipartimento di Fisica, Università di Parma and INFN Gruppo Collegato di Parma, \\ Parco Area delle Scienze, 7/A, 43100 Parma, Italy \\ E-mail: depietri@fis.unipr.it, feo@fis.unipr.it
}

\section{Erhard Seiler}

MPI für Physik - Werner Heisenberg Institut für Theoretische Physik, München, Germany

E-mail: ehs@mppmu.mpg.de

\section{Ion-Olimpiu Stamatescu}

FEST - Protestant Institute for Interdisciplinary Research, Heidelberg and Institut für Theoretische Physik der Universität, Heidelberg, Germany

E-mail: stamates@thphys.uni-heidelberg.de

\begin{abstract}
We study the high density region of QCD within an effective model obtained in the frame of the hopping parameter expansion. The model still acknowledges the sign problem peculiar to nonzero chemical potential, but it permits the development of refined algorithms which ensure a good overlap of the Monte Carlo ensemble with the true one. We review the main features of the model, including the most explicit form of the resumed expansion, and present calculations concerning the dependence of various observables on the chemical potential and on the temperature, in particular of the charge density and the diquark susceptibility, which may be used to characterize the various phases expected at high baryonic density.
\end{abstract}

XXIIIrd International Symposium on Lattice Field Theory

25-30 July 2005

Trinity College, Dublin, Ireland

${ }^{*}$ UPRF-2005-03

†Speaker.

FI-O.S. is indebted to the MPI München for hospitality and support while part of this work has been performed and to DFG for support in attending the conference. 


\section{Introduction}

The aim of this work is to understand the phase structure of high density, strongly interacting matter. In the spirit of the $\mu=0$ quenched approximation one has considered a "non-zero density quenched approximation" for $\mu>0$ based on the double limit $M \rightarrow \infty, \mu \rightarrow \infty, \zeta \equiv \exp (\mu-\ln M)$ : fixed $[1,2]$. This implements a static, charged background, which influences the gluonic dynamics $[2,3]$. The present model [4] represents a systematic extension of the above considerations: the gluonic vacuum is enriched by the effects of dynamical quarks of large (but not infinite) mass, bringing a large net baryonic charge. In [5] and in the present paper we explore the phase structure of the model, as a first step in understanding the properties of such a background.

This model can be derived as $1 / M$ expansion of QCD at large $\mu$ around the unphysical massive quarks point. However, it is more realistic to understand it as an approximation, whose justification relies on the predominant role of the gluonic dynamics. We want to understand how this dynamics is influenced by the presence of charged matter. This would allow, among other things, to study the effect of dense, heavier background baryonic charges on light quarks and hadrons.

The main ingredient of the model are Polyakov-type of loops, capturing the effect of heavy, limitedly moving quarks. The model still has a sign problem, but due to the factorization of the fermionic determinant, it allows for local, refined algorithms and large statistics.

\section{The model}

The QCD grand canonical partition function with Wilson fermions at $\mu>0$ is [6]:

$$
\begin{aligned}
& \mathscr{Z}(\beta, \kappa, \mu)=\int[D U] \mathrm{e}^{-S_{G}(\beta,\{U\})} \mathscr{Z}_{F}(\kappa, \mu,\{U\}), \mathscr{Z}_{F}(\kappa, \mu,\{U\})=\operatorname{Det} W(\kappa, \mu,\{U\}), \\
& W_{f f^{\prime}}=\delta_{f f^{\prime}}\left[1-\kappa_{f} \sum_{i=1}^{3}\left(\Gamma_{+i} U_{i} T_{i}+\Gamma_{-i} T_{i}^{*} U_{i}^{*}\right)-\kappa_{f}\left(\mathrm{e}^{\mu_{f}} \Gamma_{+4} U_{4} T_{4}+\mathrm{e}^{-\mu_{f}} \Gamma_{-4} T_{4}^{*} U_{4}^{*}\right)\right] \\
& \Gamma_{ \pm \mu}=1 \pm \gamma_{\mu}, \quad \kappa=\frac{1}{2(M+3+\cosh \mu)}=\frac{1}{2\left(M_{0}+4\right)},
\end{aligned}
$$

with $M$ the "bare mass", $M_{0}$ : bare mass at $\mu=0, f$ : flavour, $U$ : links and $T$ : lattice translations.

The hopping parameter expansion is an expansion in closed loops of Det $W=\prod_{f} \operatorname{Det} W_{f}$,

$$
\begin{aligned}
\operatorname{Det} W_{f} & =\exp \left[-\sum_{l=1}^{\infty} \sum_{\left\{\mathscr{C}_{l}\right\}} \sum_{s=1}^{\infty} \frac{\left(\kappa_{f}^{l} g_{\mathscr{C}_{l}}^{f}\right)^{s}}{s} \operatorname{Tr}_{\mathrm{D}, \mathrm{C}}\left(\mathscr{Y}_{\mathscr{C}_{l}} \mathscr{L}_{\mathscr{C}_{l}}\right)^{s}\right]=\prod_{l=1}^{\infty} \prod_{\left\{\mathscr{C}_{l}\right\}} \prod_{\alpha=1}^{4} \operatorname{Det}_{\mathrm{C}}\left(1-\left(\kappa_{f}\right)^{l} g_{\mathscr{C}_{l}}^{f} y_{\mathscr{C}_{l}}^{\alpha} \mathscr{L}_{\mathscr{C}_{l}}\right) \\
g_{\mathscr{C}_{l}}^{f} & =\left(\varepsilon \mathrm{e}^{ \pm N_{\tau} \mu_{f}}\right)^{r} \text { if } \mathscr{C}_{l}: \text { :Polyakov r-path", }=1 \text { else, } \mathscr{Y}_{\mathscr{C}_{l}}=\prod_{l \in \mathscr{C}_{l}} \Gamma_{l}, \mathscr{L}_{\mathscr{C}_{l}}=\prod_{l \in \mathscr{C}_{l}} U_{l}
\end{aligned}
$$

where $\mathscr{C}_{l}$ are distinguishable, non-exactly-self-repeating closed paths of length $l, s$ is the number of coverings of $\mathscr{C}_{l}$, and a "Polyakov $r$-path" winds $r$ times over the lattice in the \pm 4 direction with periodic(antiperiodic) b.c. $\varepsilon=+1(-1)$ (p.b.c. in spatial directions). The factors $y_{\mathscr{C}_{l}}^{\alpha}$ are the eigenvalues of $\mathscr{Y}_{\mathscr{C}_{l}}$ and the expansion can be expressed as a product of colour determinants [7]. Notice that Det $W$ is a polynomial in $\kappa$ of the order $4 n_{c} n_{f} N_{V}$ (colours, flavours, lattice volume), which means that the expansions on the right hand side can be truncated at this order. 


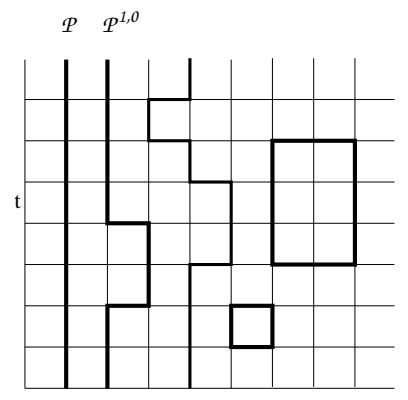

$\kappa^{0} \quad \kappa^{2}$

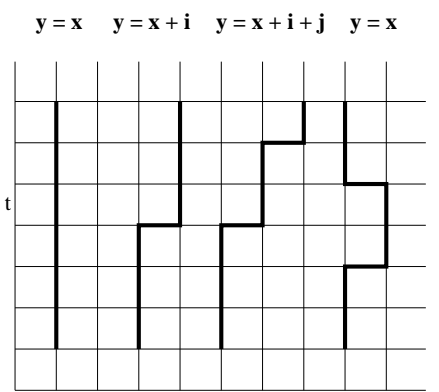

$\kappa^{2}$



$\kappa^{0}$

$\kappa^{2}$

Figure 1: Loops in the determinant (left); quark (middle) and di-quark propagators (right).

The "quenched limit at $\mu>0$ " is [1]:

$$
\begin{aligned}
& \kappa \rightarrow 0, \mu \rightarrow \infty, \kappa \mathrm{e}^{\mu} \equiv \zeta: \text { fixed, } C=(2 \zeta)^{N_{\tau}} \\
& \mathscr{Z}_{F}^{[0]}(C,\{U\})=\exp \left[-\sum_{\{\vec{x}\}} \sum_{s=1}^{\infty} \frac{(\varepsilon C)^{s}}{s} \operatorname{Tr}_{\mathrm{C}}\left(\mathscr{P}_{\vec{x}}\right)^{s}\right]=\prod_{\{\vec{x}\}} \operatorname{Det}_{\mathrm{C}}\left(1-\varepsilon C \mathscr{P}_{\vec{x}}\right)^{2},
\end{aligned}
$$

and the next order corrections lead to [4]:

$$
\begin{aligned}
\mathscr{Z}_{F}^{[2]}(\kappa, \mu,\{U\}) & =\exp \left\{-2 \sum_{\{\vec{x}\}} \sum_{s=1}^{\infty} \frac{(\varepsilon C)^{s}}{s} \operatorname{Tr}_{\mathrm{C}}\left[\left(\mathscr{P}_{\vec{x}}\right)^{s}+\kappa^{2} \sum_{r, q, i, t, t^{\prime}}(\varepsilon C)^{s(r-1)}\left(\mathscr{P}_{\vec{x}, i, t, t^{\prime}}^{r, q}\right)^{s}\right]\right\} \\
& =\mathscr{Z}_{F}^{[0]}(C,\{U\}) \prod_{\vec{x}, r, q, i, t, t^{\prime}} \operatorname{Det}_{\mathrm{C}}\left(1-(\varepsilon C)^{r} \kappa^{2} \mathscr{P}_{\vec{x}, i, t, t t^{\prime}}^{r, q}\right)^{2} .
\end{aligned}
$$

See Fig. 1. For easy bookkeeping we use the incomplete temporal gauge:

$$
\begin{aligned}
& U_{n, 4}=1, \text { except } U_{\left(\vec{x}, n_{4}=N_{\tau}\right), 4} \equiv V_{\vec{x}}, \\
& \mathscr{P}_{\vec{x}}=V_{\vec{x}}, \mathscr{P}_{\vec{x}, i, t, t^{\prime}}^{r}=\left(V_{\vec{x}}\right)^{r-q} U_{(\vec{x}, t), i}\left(V_{\vec{x}+\hat{i}}\right)^{q} U_{\left(\vec{x}, t^{\prime}\right), i}^{*}, r>q \geq 0, \pm i=1,2,3,1 \leq t \leq t^{\prime} \leq N_{\tau}
\end{aligned}
$$

$\left(t<t^{\prime}\right.$ for $q=0$ ). Eqs. (2.4-2.8) define the present model. Notice that for $U \in \mathrm{SU}(3)$ we have:

$$
\operatorname{Det}_{C}(1-\varepsilon C U)=1-\varepsilon C \operatorname{Tr} U+C^{2} \operatorname{Tr} U^{\dagger}-\varepsilon C^{3}
$$

We measure spatial and temporal plaquettes, Polyakov loops and baryon charge densities

$$
\frac{n_{B}}{T^{3}}=\frac{N_{\tau}^{3}}{3 N_{\sigma}^{3}} \hat{n}, \hat{n}=\hat{n}_{0}+\hat{n}_{1}, \quad \hat{n}_{0}=\left\langle\frac{\partial}{\partial \mu} \ln \mathscr{Z}_{F}^{[0]}\right\rangle, \quad \hat{n}_{1}=\left\langle\frac{\partial}{\partial \mu}\left(\ln \frac{\mathscr{Z}_{F}^{[2]}}{\mathscr{Z}_{F}^{[0]}}\right)\right\rangle
$$

as well as diquark propagators (in maximal temporal gauge) and the corresponding susceptibility, which could give information on the large $\mu$ physics [8] (see [5] for explicite formulae, see Fig. 1).

\section{Algorithm and Simulation}

We use the Wilson action and Wilson fermions within a reweighting procedure. The updating is done with a local Boltzmann factor (which only leads to a redefinition of the "rest plaquette"):

$$
\prod_{\text {Plaq }} \mathrm{e}^{\frac{\beta}{3} \operatorname{Tr} P l a q} \prod_{\vec{x}} \exp \left\{2 C \mathscr{R} e \operatorname{Tr}_{\mathrm{C}}\left[\mathscr{P}_{\vec{x}}+\kappa^{2} \sum_{r, q, i, t, t^{\prime}} \mathscr{P}_{\vec{x}, i, t, t^{\prime}}^{r, q}\right]\right\} \text {. }
$$


We here employ the Cabbibo-Marinari heat-bath procedure mixed with over-relaxation. This updating already takes into account part of the $\mu>0$ effects and the generated ensemble can thus have a better overlap with the true one than an updating at $\mu=0$. Notice that such a factor may also improve the convergence of full QCD simulations at $\mu>0$. The weight (global, vectorizable) is:

$$
\prod_{\vec{x}} \exp \left\{-2 C \mathscr{R} e \operatorname{Tr}_{\mathrm{C}}\left[\mathscr{P}_{\vec{x}}+\kappa^{2} \sum_{r, q, i, t, t^{\prime}} \mathscr{P}_{\vec{x}, i, t, t^{\prime}}^{r, q}\right]\right\} \mathscr{Z}_{F}^{[2]}(\{U\}) .
$$

One also can use an improved partition between the updating factor and the weight, to be taken care of by a supplementary Metropolis check. Anisotropy can be straightforwardly introduced.

The simulations are mainly done on lattices $6^{4}$ and $8^{4}$ (in the incomplete temporal gauge (2.7)). We shall present results for $n_{f}=1$ and $n_{f}=3$ degenerate flavours (any mixture of flavours can be implemented). The $\kappa$ dependence has been analyzed in [4]. Here we set $\kappa=0.12$, which drives the $1 / M^{2}$ effects in the baryonic density to about $50 \%$. Our problem setting is primordially to explore the phase structure of the model at large $\mu$, small $T$ and we accordingly vary $\beta$ and $\mu$.

\section{Results}

Some analytic results can be obtained in strong coupling. To lowest order in $\beta$ we have:

$$
\begin{aligned}
P & =\frac{1}{3} \operatorname{Tr}\left\langle\frac{1}{N_{\sigma}^{3}} \sum_{\vec{x}} \operatorname{Tr} \mathscr{P}_{\vec{x}}\right\rangle=C^{2} \frac{1+\frac{2}{3} C^{3}}{1+4 C^{3}+C^{6}}, \\
P^{\dagger} & =\frac{1}{3} \operatorname{Tr}\left\langle\frac{1}{N_{\sigma}^{3}} \sum_{\vec{x}} \operatorname{Tr} \mathscr{P}_{\vec{x}}^{\dagger}\right\rangle=\frac{2}{3} C \frac{1+C^{3}}{1+4 C^{3}+C^{6}}\left[1+\frac{2 N_{\tau}}{3} \beta \kappa^{2} \frac{1+3 C^{2}+2 C^{3}+2 C^{5}+C^{6}}{1+C^{3}}\right]
\end{aligned}
$$

for $n_{f}=1$. Fig. 2 shows increasing deviation of the full results from strong coupling with increasing $\beta$ and indication of qualitative change of behaviour at large $\mu$ and above $\beta=5.5$.
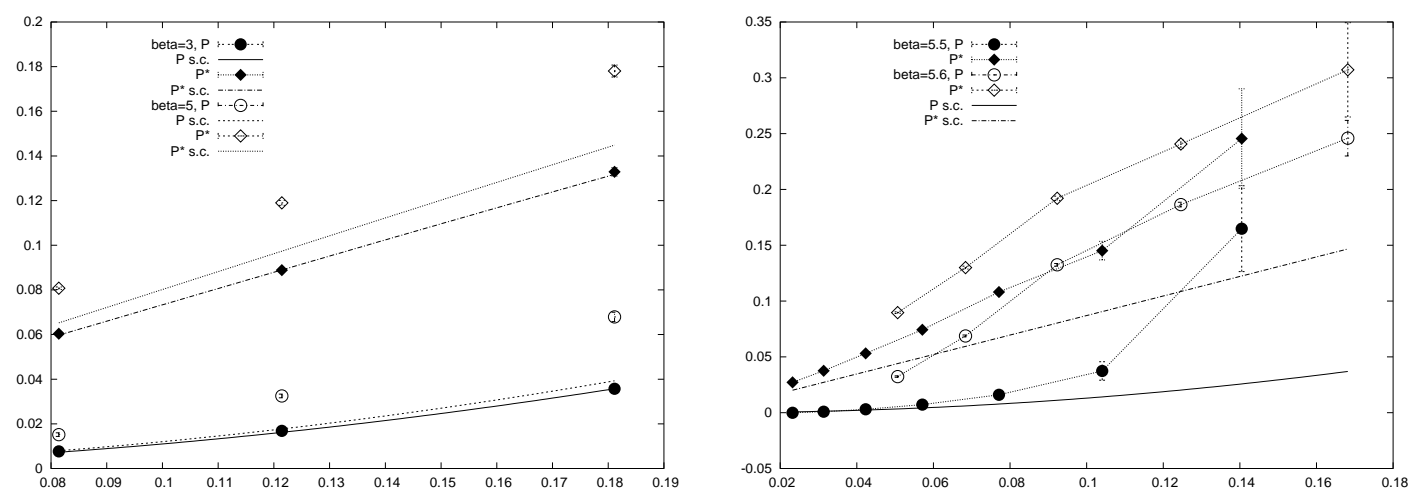

Figure 2: Polyakov loops $P$ (triangles) and $P^{\dagger}$ (diamonds) vs $C$, $4^{4}$ (left, $\beta=3$, full and $\beta=5$, open symbols) and $6^{4}$ (right, $\beta=5.5$, full symbols and $\beta=5.6$, open symbols), strong coupling (lines).

On Fig. 3, left plot, we show for $n_{f}=3$ the behaviour with $\beta$ at fixed $\mu$ values, which in the physical $T, \mu$ plane would mean moving on lines $T \simeq \frac{1}{\mu N_{\tau}} \mu_{\text {phys. }}$. We see at both values of $\mu$ qualitative changes of behaviour suggesting transitions from low to high temperature phases at 
values of $\beta$ as indicated by the Polyakov loop susceptibility (curves on the plot). This appears corroborated by the behaviour shown on the right hand plot, where we vary $\mu$ at two fixed $\beta$ values and see a delayed onset of the "transition" for the smaller $\beta$. The diquark susceptibility (we only show the second order term) is rather flat in $\beta$ and shows a signal only at $\mu$ approaching 1 .


Figure 3: Baryonic density (triangles), Polyakov loops (circles) and diquark susceptibility (squares) vs $\beta$ (left, $\mu=0.85$ open, and $\mu=0.90$ full symbols) and $v s \mu$ (right, $\beta=5.5$ full and $\beta=5.6$ open symbols).

The dependence on $n_{f}$ is illustrated on Fig. 4 , both for the $6^{4}$ and the $8^{4}$ lattices. As one can see, the highest values of $\mu$ attainable in this method are about $5 \sim 6 T$, depending on $n_{f}$.
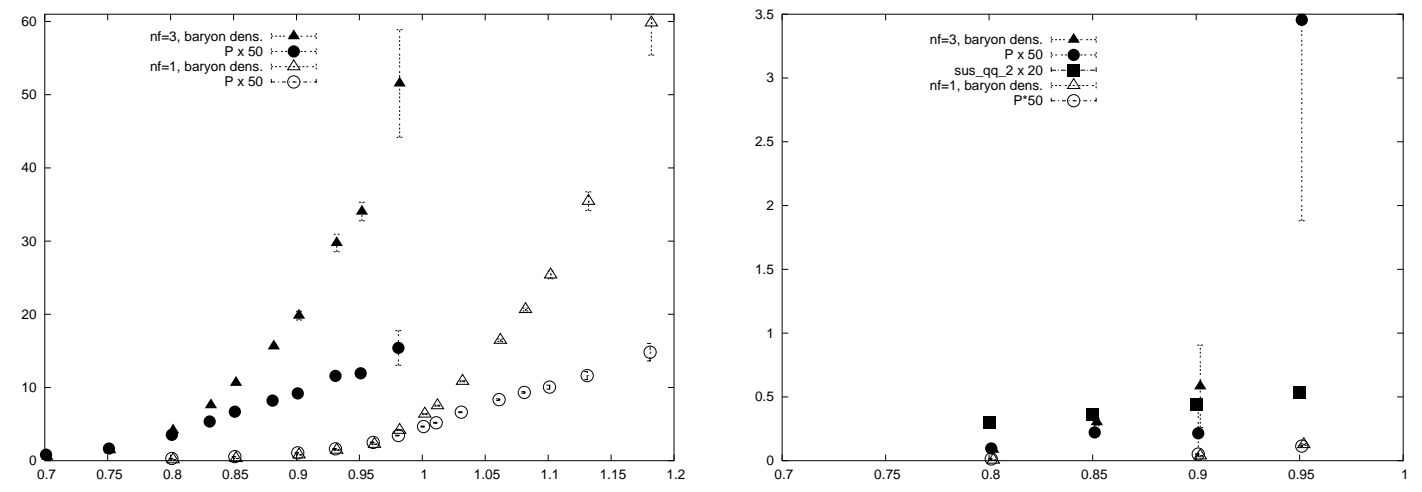

Figure 4: Baryonic density (triangles) and Polyakov loops (circles) $v s \mu$ at $\beta=5.6,6^{4}$ (left) and $8^{4}$ (right; here also the diquark susceptibility, squares): $n_{f}=1$ open and $n_{f}=3$ full symbols.

\section{Discussion}

Our calculations show strong effects at large $\mu$, which even at moderate $\beta$ depart considerably from strong coupling estimates and also indicate possible phase transitions. The results concerning the latter for the $n_{f}=3$ case are summarized in Fig. 5. On the left plot we indicate the lines we have followed in the simulation in the $T, \mu_{\text {phys }}$ plane. On the right hand plot we show the $\beta, \mu$ plane together with the points at which we see indication of transitions. Since we cannot yet fix a scale (but see below) we preferred to show these points in the bare parameter plane. While the indication for a transitions are clear, it is uncertain what happens at larger $\mu$ and small $T$ and how the transition lines run. This region needs therefore further study. 

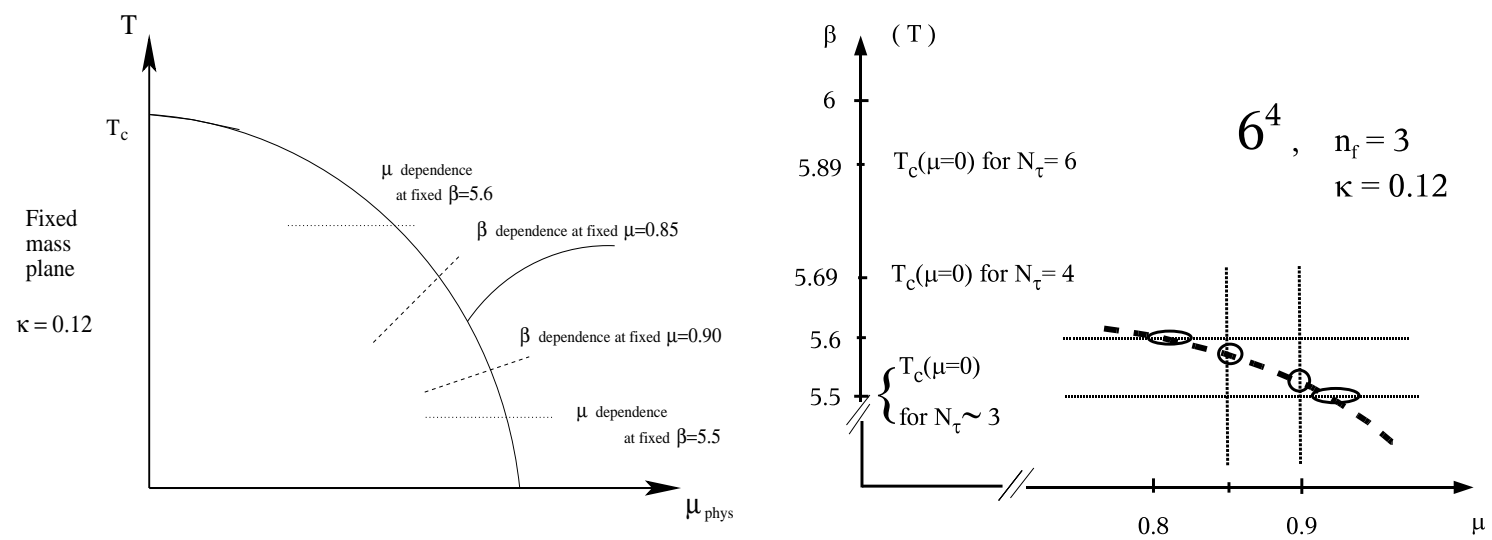

Figure 5: Prospected phase structure (only indicative!) and observed changes of behaviour (transitions?).

The algorithm works reasonably well over a wide range of parameters and for lattices up to $8^{4}$. We reach large densities and $\frac{\mu}{T}$ for temperatures $\sim \frac{1}{3} T_{c}(\mu=0)$ or less. It appears difficult, however, to go to larger lattices and larger $\mu$ with this algorithm and one should consider improving it.

The model permits to vary $\mu, \kappa$ and $T$ as independent parameters. It is therefore interesting to extend the study to cover this full variability. For higher orders in $\kappa$ the bookkeeping soon becomes unmanageable, one could however consider using statistical ensembles of large loops [9].

Concerning the significance of this analysis we can take two points of view:

Firstly, we can consider this model for itself, as describing "quasi-static charges" interacting via gauge forces. One may then ask whether this dynamics may lead to a non-trivial phase structure.

Secondly, we can consider this model as an evolved "quenched approximation" in the presence of charged matter. Then this study would give us information about the structure of the so modified gluonic vacuum of the SU(3) theory. It would then be natural to think of it as providing a heavy, dense, charged background for light quarks propagation and calculate light hadron spectra and other hadronic properties under such conditions. This could also help fixing a scale.

Acknowledgments: The calculations have been done on the VPP5000 computer of the University of Karlsruhe and on the PC Cluster of the Institute of Physics of the Parma University.

\section{References}

[1] I. Bender et al., Nucl. Phys. B (Proc.Suppl.) 26 (1992) 323.

[2] J. Engels et al., Nucl. Phys. B 558 (1999) 307.

[3] T. C. Blum, J. E. Hetrick and D. Toussaint, Phys. Rev. Lett. 76 (1996) 1019. O. Kaczmarek, Ph.D. Thesis, Bielefeld 2000; A. Yamaguchi, Nucl. Phys. B (Proc.Suppl.) 106 (2002) 465.

[4] G. Aarts et al., Nucl. Phys. B (Proc.Suppl.) 106 (2002) 456.

[5] R. Hofmann and I-O. Stamatescu, Nucl. Phys. B (Proc.Suppl.) 129 (2004) 623.

[6] P. Hasenfratz and F. Karsch, Phys. Lett. 125B (1983) 308. J. Kogut et al, Nucl. Phys. B225 (1983) 93.

[7] I.-O. Stamatescu, Phys. Rev. D25 (1982) 1130. I.-O. Stamatescu and T. T. Wu, preprint CERN-TH-6631-92 (1993).

[8] M. G. Alford, K. Rajagopal and F. Wilczek, Nucl. Phys. B537 (1999) 443.

[9] M. G.Schmidt and I.-O. Stamatescu, Mod. Phys. Lett. A18 (2003) 1499. 